\title{
A medida del cuerpo. Notas acerca del retorno a lo físico en la estética de las nuevas tecnologías*
}

\author{
According to the body. Notes on the re-turn to material world in \\ new technologies aesthetic
}

ALEJANDRO LOZANO MUÑOZ**

\begin{abstract}
Resumen: Estas notas abordan 2 puntos interconectados: 1) en primer lugar daremos cuenta de algunos de los factores clave (a nivel coyuntural, social y especialmente tecnológico) que han propiciado un retorno paralelo al cuerpo y al espacio físico. A continuación, y tomando como referencia algunos de los discursos teóricos en torno a la fisionomía actual del biopoder, 2) esbozaremos un enfoque alternativo en torno a las relaciones entre cuerpo, nuevas tecnologías y poder partiendo de prácticas ciudadanas en las que la inclusión de dichas tecnologías potencia (en lugar de socavar, gestionar y controlar) la identidad los sujetos que hacen causa común para ejercer resistencia y hacer valer sus intereses.
\end{abstract}

Palabras clave: cuerpo, nuevas tecnologías, espacio, biopoder, ciudadanía, identidad.

\begin{abstract}
This notes point towards two intertwined topics: 1) first of all, we will approach to some of the key factors (conjunctural, social and technological) regarding the simultaneous turn to both the body and the physical space. Then, taking as references different narratives about contemporary biopower, 2), we will sketch out an alternative framework concerning the bonds between body, new technologies and power. In order to do so, we will focus on recent social practices which include the use of these technologies as tools of empowerment.
\end{abstract}

Keywords: body, new technologies, space, biopower, citizenship, identity.

Fecha de recepción: 01/06/2016. Fecha de aceptación: 27/07/2016.

* Esta investigación ha sido posible gracias a un contrato predoctoral cofinanciado por la Universidad de Salamanca y el Banco Santander.

** Investigador predoctoral en la Universidad de Salamanca. Su principal línea de investigación es la estética de las nuevas tecnologías (biotecnologías, tecnologías digitales y tecnologías sanitarias) en las sociedades contemporáneas. Sus principales intereses investigadores son: a) Las relaciones entre tecnología, cuerpo y espacio en un mundo tecnificado; b) Cambios en la percepción del yo, los otros y el entorno circundante debido al uso creciente de diferentes tipos de tecnología en nuestra vida cotidiana; c) Nuevas narrativas digitales tras el discurso de la cibercultura (1980-1999).Dirección de contacto: alejandro.lm@usal.es 


\section{Retorno al cuerpo en la estética de las nuevas tecnologías tras el ocaso de las ciber- culturas}

\subsection{Contexto. El ocaso de las ciberculturas y la obsolescencia de la estética del ciberespacio}

Comprender los giros hacia el cuerpo físico y hacia los espacios locales que han tenido lugar en nuevas tecnologías durante los últimos años requiere situarse en el contexto del ocaso de las ciberculturas históricas ${ }^{1}$. Aunque se trata de un fenómeno que tiene lugar en esferas como la artística, la científica o la filosófica, es especialmente la literatura el medio más influyente para generar la estética de las nuevas tecnologías caracteristica del periodo de las ciberculturas. Durante los años 80 y 90 del siglo pasado aparecieron casi de manera sistemática varias obras de ciencia-ficción que sentarían las bases de una estética que todavía hoy ejerce un notable influjo. Nos referimos a novelas fundacionales del cyberpunk como Neuromante (1984) de William Gibson, o a la compilación Mirrorshades (1986) editada por Bruce Sterling, cuyo prólogo establece las preocupaciones fundamentales de este subgénero de la ciencia ficción. En este eje de coordenadas surge el ciberespacio, desbordando con su inconcebible estructura la imaginación del lector ${ }^{2}$.

Aunque la cibercultura en su conjunto (particularmente la subcultura ciberpunk) había mantenido desde el comienzo una relación problemática con el cuerpo, se puede considerar a Stelarc como una de las figuras que, desde el ámbito de la performance artística, ha expresado con mayor rotundidad la situación de nuestra dimensión orgánica en aquel contexto. En una de sus citas más conocidas el artista chipriota, conocido por sus performances en torno a la transformación del cuerpo humano por parte de la tecnología, sostiene:

Es hora de preguntarse si un cuerpo bípedo, que respira, y que posee una visión binocular y un cerebro de 1400 centímetros cúbicos es una forma biológica adecuada. Ya no da abasto debido a la cantidad, complejidad y calidad de la información acumulada... La información propele el cuerpo más allá de sí mismo y de su biosfera. La información determina la naturaleza y la función del cuerpo postevolutivo (Dery, 1998, p. 183).

1 Roy Ascott definió concisamente la cibercultura como "el dominio de aparición en el que la inteligencia natural y la vida artificial pueden interactuar creativamente (Ascott, 2007: 279). Una conceptualización más técnica es la que ofrecen Sánchez Perera y Andrada de Gregorio: "Diversos autores denominan "cibercultura", a la agrupación de una serie de fenómenos culturales contemporáneos ligados principal, aunque no únicamente, al profundo impacto que han venido ejerciendo las tecnologías digitales de la información y la comunicación sobre aspectos tales como la realidad, el espacio, el tiempo; el humano mismo y sus relaciones sociales" (Sánchez Perera \& Andrada de Gregorio, 2013). También es pertinente, pese a que se ciña al ámbito ciberpunk, la descripción que ofrece Sterling de la problemática de esta subcultura: "Ciertos temas centrales aparecen con frecuencia en el ciberpunk: el problema de la invasión del cuerpo con miembros protésicos, circuitos implantados, cirugía plástica o alteración genética. Similar y quizás aún más poderosa es la invasión de la mente: interfaces mente-ordenador, inteligencia artificial, neuroquímica... son técnicas que redefinen radicalmente la naturaleza humana, la naturaleza del yo" (Sterling, 1998).

2 Es conocido el siguiente párrafo de la novela seminal de Gibson en el que se describe el nuevo espacio inmaterial con una terminología que recuerda a las experiencias con drogas: "El ciberespacio. Una alucinación consensual experimentada diariamente por billones de legítimos operadores, en todas las naciones, por niños a quienes se enseña altos conceptos matemáticos... Una representación gráfica de la información abstraída de los bancos de todos los ordenadores del sistema humano. Una complejidad inimaginable" (Gibson, 2010: 69-70). 
Otro texto sumamente influyente fue Pigs in cyberspace (1992) del especialista en robótica Hans Moravec, quien, en la órbita del transhumanismo y a través de un relato especulativo sobre el futuro de la realidad virtual y el ciberespacio, llegó a afirmar lo siguiente:

Eventualmente, quizá queramos superar nuestros músculos atrofiados y nuestros sentidos atenuados si la neurobología aprende lo suficiente como para conectar nuestros nervios sensores y motores a interfaces electrónicas (Moravec, 2013: 179).

Las décadas de las ciberculturas se caracterizaron así por un fuerte dualismo que separaba netamente la realidad material de la realidad electrónica y líquida de los espacios electrónicos. En el peor de los casos el cuerpo quedaba condenado al eventual abandono tras la adopción de una vida cada vez más adaptada al ritmo del universo digital, o bien a la obsolescencia como víctima del upgrade tecnológico. Rara vez se llamó la atención de la fuerza con la que este dualismo volvía a separar la carne de la mente.

Las utopías y distopías de las ciberculturas han ido desvaneciéndose a medida que avanzaba el nuevo milenio debido a una serie de factores coyunturales (hitos como el bluff del efecto 2000 o la crisis de las empresas punto com), sociales (la asimilación cada vez mayor de las nuevas tecnologías en la vida cotidiana de los usuarios, eliminando su carácter de novum o acontecimiento revolucionario), y particularmente tecnológicos acerca de los que es conveniente detenerse.

\subsection{Contexto tecnológico. Conectividad y portabilidad como factores tecnológicos del giro hacia el cuerpo (y hacia el espacio).}

En el momento presente se puede constatar una metamorfosis en la estética de las nuevas tecnologías en la que el cuerpo físico ha adquirido un papel protagonista. Cabe señalar la emergencia de un nuevo paradigma tecnológico caracterizado por la conectividad, la movilidad y la integración de los dispositivos con el espacio físico que se aleja de la concepción de las tecnologías digitales como portales de acceso a un nuevo espacio electrónico.

A continuación señalamos una serie de innovaciones tecnológicas que han actuado como factores materiales determinantes para esta transformación:

1) La rápida acogida en las sociedades desarrolladas de una serie de dispositivos tecnológicos aparecidos a finales de la década de los 2000 (smarthones, tablets, relojes inteligentes, wearables) que trajeron consigo una serie de innovaciones en términos de diseño: conectividad permanente, portabilidad y, como consecuencia, facilidad de uso durante todo tipo de desplazamientos.

2) El desarrollo de redes de Banda Ancha Móvil (2G, 3G, 4G) que posibilitan un acceso prácticamente ubicuo e instantáneo a Internet, suprimiendo la necesidad de recurrir a equipos fijos como el ordenador de sobremesa para acceder a la red (IHS Inc. \& Valdani Vicari \& Associati, 2014; Ministerio de industria, Energía y Turismo, 2014).

3) Ligado a lo anterior, la presencia de tecnología GPS, sensores de movimiento (acelerómetros) y rotación (giroscopios) en estos dispositivos favorece un uso localizado de las nuevas tecnologías. Sirviendose de estos sistemas han aparecido 
numerosas aplicaciones que se adaptan para ajustar la experiencia del usuario al espacio en el que se encuentra (Foursquare, Google Maps, aplicaciones deportivas como Endomondo, etc.).

4) Por último, la consolidación de la interfaz táctil como la mediadora entre usuario y sistema ha permitido la integración del sentido del tacto en la estética de las nuevas tecnologías, acercando físicamente a los dispositivos al cuerpo de los usuarios (Lozano, 2014).

Esta coyuntura ha propiciado un cambio de orientación en nuestra relación con las tecnologías digitales. En ella el cuerpo juega ahora un renovado papel, lejos ya de la problemática planteada por la estética del ciberespacio, y favorece una percepción háptica y localizada de las mismas. Por háptico no hay que entender únicamente la presencia del tacto de la mano, sino también del movimiento y, en general, de los múltiples mecanismos mediante los que nuestro organismo capta toda clase de estímulos a través de la piel: "La percepción háptica se define usualmente como la combinación de funciones táctiles, kinestésicas y propioceptivas, el modo en que experimentamos el tacto tanto en la superficie como en el interior de nuestros cuerpos" (Richardson \& Wilken, 2009: 29). Técnicamente, la háptica es "un sistema perceptivo, mediado por dos subsistemas aferentes, cutáneo y kinestésico, que involucra habitualmente la exploración manual activa [...] El sistema háptico es especialmente efectivo a la hora de procesar las características materiales de superficies y objetos" (Lederman \& Klatzky, 2009: 1439).

El cuerpo aparece no ya como la carne residual o la mitad prescindible del sujeto, sino como la piedra de toque, a la manera del cuerpo "aquí" o del "espacio corpóreo" de MerleauPonty $^{3}$ desde el que habitar y percibir un entorno en el que lo físico y lo digital forman parte de la misma realidad. Cada vez más las nuevas tecnologías se diseñan con el objetivo de sacar mayor partido de algunas de las posibilidades de nuestro organismo, como la háptica, la frecuencia cardiaca o simplemente la capacidad de desplazamiento ${ }^{4}$. En este sentido el cuerpo físico, con sus posibilidades y limitaciones, se está convirtiendo en unidad de medida de las nuevas tecnologías. Este retorno a la materialidad del organismo confirma el ocaso de las utopías digitales una vez comprobada la inviabilidad de las múltiples promesas que confiaban al devenir del desarrollo tecnológico una vida mejor sin los obstáculos de la carne.

Desde el punto de vista de la estética y apoyándose en la ciencia, hay que ir más allá de la reducción de la sensibilidad a los denominados "cinco sentidos". La idea del cuerpo como punto de partida, así como la importancia central que la dimensión física adquiere en

3 "La palabra «aquí», aplicada a mi cuerpo, no designa una posición determinada con respecto a otras posiciones o con respecto a unas coordenadas exteriores, sino la instalación de las primeras coordenadas, el anclaje del cuerpo activo en un objeto, la situación del cuerpo ante sus tareas. El espacio corpóreo es [...] la zona de no-ser ante la cual pueden aparecer unos seres precisos, figuras y puntos" (Merleau-Ponty, 1993).

4 Se puede mencionar, en este sentido, el incipiente movimiento del "yo cuantificado" (quantified self), una corriente impulsada desde 2007 por Kevin Kelly y Gary Wolf (Kelly \& Wolf, 2007). La filosofía del movimiento QS se centra en el individuo y en la percepción de la propia identidad y considera que plasmar determinadas facetas del estilo de vida en herramientas de registro y visualización conduce a un conocimiento más profundo y veraz de quién se es realmente. En una deriva hacia el fitness del "cuidado del ser" (Swan, 2013), cada vez más usuarios consultan en retrospectiva las actividades que les interesa controlar para conocer con datos cuantitativos numerosos aspectos de sus vidas. 
el pensamiento de Merleau-Ponty,, encuentra eco en Embodied Mind, el tratado de ciencia cognitiva ya clásico de Varela (Varela, Thompson, \& Rosch, 1993). En la misma línea de trabajo se encuentran las argumentaciones de Damasio desde la neurociencia, que exhorta a comprender al "cuerpo como norma" insoslayable para percibir el entorno que nos rodea: "El organismo mismo, y no alguna realidad absoluta externa, es usado como fundamento y referencia de nuestra construcción del mundo circundante y de la construcción del omnipresente sentido de subjetividad que es parte esencial de nuestras experiencias" (Damasio, 1999: 16). También en los 90 Lakoff y Johnson, con su concepción de un realismo encarnado, presentaron en Philosophy in the Flesh la hoja de ruta para pensar al cuerpo como un componente fundamental en todos los procesos humanos, incluyendo la formación de conceptos, al afirmar que la percepción de la realidad depende estrechamente del aparato sensomotor de los individuos, que a la postre es el que los capacita para percibir y manipular objetos, moverse, etc. (Lakoff \& Johnson, 1999).

Siguiendo estas propuestas, parece imperativo concebir al individuo en su conjunto como un cuerpo inteligente y sensible, un productor de experiencias poliestéticas que no percibe una imagen solo con la vista al igual que no recorre una calle únicamente con los pies. No obstante, y desde un punto de vista crítico, hay que señalar que este retorno a lo físico no está exento de dificultades. Si bien es cierto que el cuerpo se está consolidando como la medida de las nuevas tecnologías, también es evidente que se privilegia un modelo de cuerpo que deja en una posición de desventaja a aquellos que no encajan en ese patrón (Ellis \& Kent, 2013). Dicho cuerpo tiene como rasgos distintivos el gozar de plena funcionalidad en el uso de manos, así como el estar dotado de vista y audición. Cabría destacar también el requisito casi indispensable de tener un buen dominio del idioma inglés (sin olvidar el protagonismo de países asiáticos como Corea del Sur). La caricatura podría completarse añadiendo que este cuerpo, presumiblemente joven y con buena salud, encontrará enormes ventajas si además es aficionado al ejercicio (especialmente si le gusta correr) y frecuenta las redes sociales digitales, lleva una vida profesional activa y realiza numerosos desplazamientos.

Por último, el uso y acceso a Internet mediante dispositivos que enfatizan la portabilidad y la interacción durante los desplazamientos ha favorecido que la movilidad se constituya como una nota característica de la actual estética de las nuevas tecnologías ${ }^{5}$. Al formar parte del flujo de actividades que se llevan a cabo en el día a día, sin necesidad de dedicar un lugar (el despacho, la sala de estar) o un tiempo específico a utilizar Internet, se desvanece la percepción de las nuevas tecnologías como portales de acceso al ciberespacio. De hecho, la noción misma de "acceso" se vuelve problemática. En su lugar, cabría decir que en estos momentos el uso de las nuevas tecnologías tiene lugar en el espacio, y no para acceder al ciberespacio ${ }^{6}$. Una vez que la realidad virtual ha cedido protagonismo a otras tecnologías de

5 La importancia que adquiere el factor móvil en este contexto ha de conectarse con el giro que han advertido investigadores en otros ámbitos como el sociológico, destacando la importancia que ha adquirido la movilidad espacial (de personas, objetos, información y capital) en la dinámica de las sociedades desarrolladas contemporáneas, un fenómeno que hasta ahora había permanecido en un lugar secundario (Sheller, 2014: 3).

6 Las generaciones más jóvenes, acostumbradas a utilizar dispositivos distintos al ordenador de sobremesa, no parecen sentir la necesidad de distinguir entre la realidad material y la realidad digital. Por ejemplo, el caso de los jóvenes japoneses, "en lugar de centrarse en asuntos como la inmersión y la creación de identidad en mundos virtuales, los usuarios son más proclives a preocuparse acerca de cómo su keitai [teléfono móvil] puede ayudarles en los espacios físicos” (De Souza e Silva, 2006: 264). 
imagen digital como la realidad aumentada, aparecen nociones tentativas que tratan de dar cuenta de la actual estética del espacio: espacios híbridos, realidad mixta, realidad aumentada o, en mi caso, realidad integrada para enfatizar la integración entre lo físico y lo digital en una realidad compleja.

\section{Tecnologías ciudadanas a partir del cuerpo. Una propuesta para abordar la comple- jidad de las relaciones entre poder, nuevas tecnologías y cuerpo}

El planteamiento hasta ahora expuesto desea expresar el cambio de sensibilidad en nuestra relación con las nuevas tecnologías (tecnologías que son ahora tanto digitales como biológicas) una vez la estética de la cibercultura ha quedado obsoleta tras el ocaso de las utopías y las distopías digitales (Molinuevo, 2006). La revalorización del cuerpo físico, que no está exenta de dificultades (puesto que se privilegia un modelo de cuerpo concreto), discurre paralea a una integración cada vez mayor de la tecnología en nuestra vida cotidiana que afecta en igual medida al espacio que habitamos. Una explicación actualizada de la estética de las nuevas tecnologías requiere una mirada atenta a casos específicos en los que se aprecien los términos de la relación actual entre sujetos y máquinas.

El doble giro (hacia lo local y hacia el cuerpo físico) en la estética de las nuevas tecnologías puede permitirnos articular una propuesta teórica que ayude a pensar las relaciones entre poder, nuevas tecnologías y cuerpo en un contexto en el que de las prácticas ciudadanas que cada vez tienen mayor importancia para configurar el espacio público. La óptica del biopoder se ve desbordada ante un panorama en el que la tecnología ya no puede pensarse únicamente como repertorio de técnicas para gestionar la vida humana. Hay que prestar atención a una serie de iniciativas que muestran cómo esa misma tecnología puede incorporarse en acciones de resistencia y ayudar a hacer valer la identidad e intereses de comunidades de afectados frente a las intenciones de terceros.

Concretamente, las nuevas tecnologías pueden formar parte iniciativas locales de tipo social y cultural de varias formas. En primer lugar, son de utilidad como herramientas auxiliares para expandir la difusión de las actividades, de modo que hagan llegar las convocatorias y llamadas a la participación a todos los usuarios de Internet. Esto permite atraer a agentes ajenos al espacio local pero que se sienten implicados con las actividades y reivindicaciones que se están llevando a cabo. El objetivo no es solo informar o abrir espacios de trabajo digitales, sino convocar a los interesados y afectados en un sitio concreto en un momento específico.

Pero, además, estas herramientas pueden ser parte de la acción misma, dotando a la comunidad de formas de expresión que no serían posibles si se careciese de estas herramientas. Esto lo ejemplifican las iniciativas artísticas de barrios como el Cabanyal, que integran recursos materiales y digitales para potenciar las reivindicaciones de los vecinos. La ciudadanía activa y preocupada por la conservación y el desarrollo desde dentro de los espacios en los que residen está comenzando a extraer todo el potencial de las nuevas tecnologías para la acción.

En esta clase de prácticas se comprueba el giro de las tecnologías de la información a las tecnologías ciudadanas, en tanto que los nuevos medios se ponen al servicio de las acciones que llevan a cabo los colectivos sociales. Una sociedad ya habituada a utilizar la infinitud 
de canales informativos disponibles a través de Internet y familiarizada con la dinámica de las nuevas tecnologías ha comenzado a utilizarlas de manera activa e innovadora en sus movilizaciones, pasando de la información a la acción.

Así, a modo de conclusión, si desde el punto de vista de la lógica del biocapitalismo el cuerpo está condenado al inmovilismo y la gestión de su inteligencia y afectos por parte de terceros, las alternativas, que surgen de las iniciativas ciudadanas con nuevos medios, muestran un cuerpo en movimiento, es decir, un cuerpo que actúa de manera autónoma sirviéndose de la misma tecnología que desde ciertos enfoques amenazan con controlarle irremediablemente.

Las nuevas tecnologías o tecnologías digitales no son ya nuevas en absoluto. Especialmente en las sociedades desarrolladas nos encontramos plenamente habituados a tratar a diario con esta clase de dispositivos, ya sea en nuestra vida cotidiana en la vida laboral, atendiendo a trámites administrativos, etc. En este sentido, es posible complementar la concepción disciplinaria de las tecnologías y las nuevas tecnologías característica de los discursos del biocapitalismo con el discurso alternativo que emana de la resistencia de una ciudadanía que se ha visto enormemente potenciadas mediante el uso de las mismas tecnologías.

\section{Referencias}

Ascott, R. (2007). Telematic Embrace. Visionary Theories of Art, Technology, and Consciousness by Roy Ascott. (E. Shanken, Ed.). Berkeley: University of California Press.

Damasio, A. (1999). El error de Descartes. Santiago de Chile: Andrés Bello.

De Souza e Silva,A. (2006). From Cyber to Hybrid Mobile Technologies as Interfaces of Hybrid Spaces. Space and Culture, 9(3), 261-278. http://doi.org/10.1177/1206331206289022

Dery, M. (1998). Velocidad de escape. La cibercultura del fin del siglo. Madrid: Siruela.

Ellis, K., \& Kent, M. (2013). Disability and New Media. New York: Routledge.

Gibson, W. (2010). Neuromante. Barcelona: Minotauro.

IHS Inc., \& Valdani Vicari \& Associati. (2014). Broadband Coverage in Europe 2013. Mapping progress towards the coverage objectives of the Digital Agenda. European Commission. Directorate-General of Communications Networks, Content \& Technology. Recuperado a partir de http://ec.europa.eu/newsroom/dae/document.cfm?doc_id=8238

Kelly, K., \& Wolf, G. (2007). What is the Quantified Self? [Web]. Recuperado a partir de http://quantifiedself.com/2007/10/what-is-the-quantifiable-self/

Lakoff, G., \& Johnson, M. (1999). Philosophy in the Flesh: The Embodied Mind and Its Challenge to Western Thought. New York: Basic Books.

Lederman, S. J., \& Klatzky, R. L. (2009). Haptic perception: a tutorial. Attention, Perception \& Psychophysics, 71(7), 1439-1459. http://doi.org/10.3758/APP.71.7.1439

Lozano, A. (2014). La digitalización del cuerpo en los smartphones. Fedro. Revista de Estética y Teoría de las Artes, 13, 31-41.

Merleau-Ponty, M. (1993). Fenomenología de la percepción. Barcelona: Planeta-Agostini.

Ministerio de industria, Energía y Turismo. (2014). Cobertura de Banda Ancha en España en el primer Trimestre de 2014. Recuperado a partir de http:/www.minetur.gob.es/telecomunicaciones/banda-ancha/cobertura/Documents/Cobertura-BA-1Trimestre2014.pdf 
Molinuevo, J. L. (2006). La vida en tiempo real. La crisis de las utopías digitales. Madrid: Biblioteca Nueva.

Moravec, H. (2013). Pigs in Cyberspace. En M. More \& N. Vita-More (Eds.), The Transhumanist Reader (pp. 177-182). Chichester: Wiley-Blackwell.

Richardson, I., \& Wilken, R. (2009). Haptic vision, footwork, place-making: a peripatetic phenomenology of the mobile phone pedestrian. Second Nature, 2, 22-41.

Sánchez Perera, P., \& Andrada de Gregorio, G. (2013). Dispositivos, prótesis y artefactos de la subjetividad cíborg. Revista de Estudios de Juventud, 102, 41-54.

Sterling, B. (Ed.). (1998). Mirrorshades. Una antología ciberpunk. Madrid: Siruela.

Swan, M. (2013). The Quantified Self: Fundamental Disruption in Big Data Science and Biological Discovery. Big Data, 1(2), 85-99. http://doi.org/10.1089/big.2012.0002

Varela, F., Thompson, E., \& Rosch, E. (1993). The Embodied Mind: Cognitive Science and Human Experience. Cambridge: The MIT Press. 